pronounced $\langle 210$ > fibre texture with the orientation axis parallel to the rod axis), and in addition strong sharp rings indicating randomly orientated beryllium oxide crystals of at least $500 \mathrm{~A}$. in diameter. Such oxide was found repertedly after re-surfacing in the manner described.

When preparing single-crystal surfaces in this way, clear Kikuchi-line and spot patterns were obtained with no sign of sharp diffractions from oxide. Thus the oxide observed on the polycrystalline surface was not produced as a result of the method of surfacing. Clearly, therefore, in the case of the hot-extruded material described above, the preferential solution of the beryllium by the etchant must have left the oxide crystals projecting above the metal, thus increasing their relative contribution to the diffraction pattern.

Further investigations, particularly of the penetration of oxygen into beryllium at high temperatures and the existence of oxide films in coarse and finegrain vacuum-cast metal, are in progress.

We wish to thank Mr. G. C. Ellis and Dr. A. Moore for the provision of specimens and for helpful discussions. One of us (V.D. Scott) is on detached duty from the Atomic Weapons Research Establishment, Aldermaston; we are indebted to the Director of this Establishment for permission to publish these results.

$$
\text { V. D. Scotr }
$$

H. WILMAN

Applied Physical Chemistry of Surfaces Laboratory, Chemical Engineering Department,

Imperial College of Science and Technology, London, S.W.7.

$$
\text { Feb. } 22 .
$$

1 Sloman, H. A., J. Inst. Metals, 49, 365 (1932).

'Kroll, W., Metallwirtschaft, 13, 725 (1934); Metal Indust., 47, 29 (1935); Metals and Alloys, 8, 349 (1937). Kaufmann, A. R. (1950). serr, I. S., and Wilman, H., J. Inst. Metals, 84, 379 (1955-56).

- Mott, B. W., and Haines, H. R., J. Inst. Metals, 80, 629 (1951-52).

\section{Kinetics of the Trigonal-to-Cubic Transition in Crystals of Cæsium Nitrate on Muscovite}

$I_{T}$ is well known ${ }^{1}$ that the epitaxial orientation of cubic cæsium nitrate on muscovite is

$\mathrm{CsNO}_{3}(100)[010]=$ muscovite $(001)[010]$

whereas the orientation of trigonal crsium nitrate is $\mathrm{CsNO}_{3}(1121)[10 \mathrm{T0}]=$ muscovite $(001)$ [010], [130]

$$
\text { and }[1 \overline{3} 0]
$$

From a general point of view, the possibility of obtaining definite orientations on definite crystallographic planes means that, of the many possible arrangements, there are some for which nucleation is more probable. In the language of statistical thermodynamics, this probability is $p=\exp \left(-\frac{\Delta F}{k T}\right)$. Consequently nucleation which gives rise to epitaxial crystals corresponds to a value of $\Delta F$ less than that for other possible arrangements. The interface between epitaxial crystals and the substrate crystal presents a smaller discontinuity than any other possible arrangement. In other words, this smaller discontinuity corresponds to both a smaller interfacial free energy and a smaller elastic strain energy.

Any polymorphic transformation undergone by the oriented crystals will involve an increase in this free energy. A shift of the equilibrium temperature, with respect to that of non-epitaxial crystals, may be foreseen. If the transformation takes place between phases of which only one is epitaxial, the shift will be large. If both phases are epitaxial, the shift will be in general smaller, and its direction will indicate the more stable epitaxy.

We have made simultaneous observations of the kinetics of the polymorphic transformation of cæsium nitrate from trigonal to cubic, on crystals of the same size obtained by evaporation of the same solution, (i) on a glass substrate and (ii) on a crystalline substrate (muscovite), in order to correlate the two processes. The two supports were placed in a Weigand heating apparatus ${ }^{2}$ fitted to a Leitz microscope, so that the crystals oriented on the mica and the nonoriented crystals on the glass were in view simultaneously. The temperature of transformation was clearly defined by the disappearance (or appearance) of birefringence on heating (or cooling) uniformly (2 deg. C. per min. at first). We have established, in many experiments, that on heating, the birefringence appears first in the crystals on the glass substrate, and on cooling it appears later. The interval between the appearance of the birefringence in the two cases has never been more than 45 sec.

The difference in behaviour described above is quite small in comparison with that observed in other experiments. For example, for the transformation $\mathrm{NaBr} \rightarrow \mathrm{NaBr} .2 \mathrm{H}_{2} \mathrm{O}$, in which only one phase $(\mathrm{NaBr})$ is epitaxial on galena, the time difference with respect to sodium bromide unoriented on glass is almost two hours. In this case the hydration brings about an appreciable increase of the interfacial discontinuity. In the case of cæsium nitrate, however, the interfacial discontinuity varies little from one phase to the other. The direction of the variation indicates that the epitaxy of the trigonal phase is more stable and more probable.

We have recently shown ${ }^{3}$ that the preponderant factor in determining the interfacial discontinuity is the amount of difference in symmetry. For cæsium nitrate, the change in the dissymmetry is almost negligible and shift in equilibrium is minimal.

\section{E. FERRONI}

M. COCCHI

Institute of Physical Chemistry, University of Florence.

1 Ferroni, E., and Cocchi, M., Z. Kristallogr. (in the press).

Angew. Chemie, 49, 243 (1936).

- Cocchi, M., and Ferroni, E., Z. phys. Chem. (in the press).

\section{An Electronic Mechanism of Addition Reactions}

A RECENT communication from Mr. Lakhbir Singh" seems to require a reply based rather more firmly on conventional theoretical chemistry. He refers to the decrease in reactivity of acetylene compared to ethylene, and implies that this shows that an increase in the number of $\pi$-bonds leads consequently to a decrease in reactivity. Duchesne ${ }^{2}$ has, however, pointed out that the greater reactivity of ethylene compared to acetylene is due to the fact that the torsional oscillations in ethylene uncouple the $\pi$-electrons, whereas this cannot happen in acetylene. Singh also describes allene as "very much strsined". It is difficult to see why this should be so, unless reliance is placed on the Baeyer ${ }^{3}$ strain theory. In modern terms the central atom may be described as having two $\sigma$-bonds in an $s p$-hybrid, with two $p$-orbitals available to form two $\pi$-bonds, the planes 